\section{G-CSF nach schwerer Neutropenie hilfreich}

\begin{abstract}
Neutropenie führt bei der Chemotherapie des Mammakarzinoms oft zu Verzögerungen oder Dosisreduktionen. In einer britischen Studie wurde untersucht, ob die Sekundärprophylaxe mit Granulozytenkolonie-stimulierenden Faktoren (G-CSF) hilft, die geplante Dosisintensität einzuhalten.
\end{abstract}

\begin{abstract}
A der randomisierten Studie nahmen
Patientinnen mit Mammakarzinom teil, die verschiedene adjuvante und neoadjuvante, nicht dosisintensivierte Chemotherapien erhielten. Verglichen wurde der Effekt von einer G-CSF-Gabe und dem Standardvorgehen nach erstmalig auftretendem neutropenischem Ereignis (NE). Definiert war dies als Hospitalisierung wegen febriler Neutropenie oder eine Neutrophilenzahl von $\leq 1.500 / \mu \mathrm{l}$ und dadurch eine Therapieverzögerung oder Reduktion der geplanten Dosis um $\geq 5 \%$. Filgrastim ( $5 \mu \mathrm{g} / \mathrm{kg}$ ) wurde von Tag
\end{abstract}

3-9 nach Ende der Chemotherapie gegeben, Pegfilgrastim (6 mg) 1-mal 24 Stunden nach Chemotherapie.

Von den geplanten 815 Patentinnen konnten nur 407 im vorgesehenen Zeitraum eingebracht werden. Bei konventionellem Vorgehen nach erstem NE (meist in einem der ersten 3 Zyklen) erreichten nur $50 \%$ der Patientinnen mindestens $85 \%$ der geplanten relativen Dosisintensität (RDI), im G-CSF-Arm $75 \%$ $(p<0,0001)$. Eine weitere schwere Neutropenie nach Randomisierung erlitten $65,7 \%$ der Patientinnen im Kontrollarm, aber nur 18,2\% im G-CSF-Arm. Unterschiede in der Toxizität zeigten sich nicht. Eine RDI von $\geq 85 \%$ erreichten $69,5 \%$ der mit Filgrastim und $84,9 \%$ der mit Pegfilgrastim behandelten Patientinnen. Der Anteil erneuter schwerer Neutropenien lag mit $22,4 \%$ versus $11 \%$ unter Filgrastim höher als unter Pegfilgrastim.

Fazit: Die G-CSF-Gabe nach erstem NE führt bei Brustkrebspatientinnen mit wenig intensiver Chemotherapie signifikant häufiger zu einer adäquaten Dosisintensität. Das dürfte sich auch auf klinische Endpunkte auswirken. Friederike Klein

Leonard RCF et al. A randomised trial of secondary prophylaxis using granulocyte colony-stimulating factor (,SPROG' trial) for maintaining dose intensity of standard adjuvant chemotherapy for breast cancer by the Anglo-Celtic Cooperative Group and NCRN. Ann Oncol. 2015;26(12):2437-41.

\section{Häufiges Problem: Orale Beschwerden}

\section{Orale Beschwerden wie Mundtrockenheit, Mukositis oder Dysphagie können die Lebensqualität von Krebspatienten stark beeinträchtigen. Nun wurde analysiert, wie oft sie bei fortgeschrittenen Erkrankungen auftreten.}

$\mathrm{M}$ ultizentrisch wurden 669 Patienten mit fortgeschrittener Krebserkrankung rekrutiert, deren Vortherapien u. a. eine Chemotherapie (15,4\%), zielgerichtete Behandlungen $(8,8 \%)$ und Palliative

Care $(71,3 \%)$ umfassten. $6,4 \%$ und $3,9 \%$ hatten eine Radiotherapie oder Operation im Kopf-Halsbereich erhalten. 149 $(22,3 \%)$ Patienten litten an Mukositis. Bei $10,7 \%$ von ihnen war sie vom WHO-

Tab. 1: Assoziationen oraler Beschwerden

\begin{tabular}{|c|c|c|c|}
\hline \multirow[b]{2}{*}{ Mukositis } & \multicolumn{3}{|c|}{ Mundtrockenheit } \\
\hline & Nein & $\mathrm{Ja}$ & $\mathrm{p}$ \\
\hline Nein & $347(66,9 \%)$ & $172(33,1 \%)$ & 0,00000 \\
\hline \multirow[t]{2}{*}{ Ja } & $51(34,2 \%)$ & $98(65,8 \%)$ & \\
\hline & \multicolumn{3}{|l|}{ Dysphagie } \\
\hline Mukositis & Nein & Ja & $\mathrm{p}$ \\
\hline Nein & $459(88,4 \%)$ & $60(11,6 \%)$ & \\
\hline \multirow[t]{2}{*}{ Ja } & $106(71,1 \%)$ & $43(28,9 \%)$ & 0,00000 \\
\hline & \multicolumn{3}{|l|}{ Dysphagie } \\
\hline Mundtrockenheit & Nein & $\mathrm{Ja}$ & $\mathrm{p}$ \\
\hline Nein & 364 (91,5 \%) & $34(8,5 \%)$ & \\
\hline Ja & 201 (74,4 \%) & $69(25,6 \%)$ & 0,00000 \\
\hline
\end{tabular}

